\title{
Tuorla quasar monitoring ${ }^{\star}$
}

\section{Observations of $1995-1997$}

S. Katajainen ${ }^{1}$, L.O. Takalo ${ }^{1}$, A. Sillanpää ${ }^{1}$, K. Nilsson ${ }^{1}$, T. Pursimo ${ }^{1}$, M. Hanski $^{1}$, P. Heinämäki ${ }^{1}$, E. Kotoneva $^{1}$, M. Lainela ${ }^{1}$, P. Nurmi ${ }^{1}$, H. Pietilä ${ }^{1}$, R. Rekola ${ }^{1}$, A. Riehokainen ${ }^{1}$, P. Teerikorpi ${ }^{1}$, E. Valtaoja ${ }^{1,2}$, and A. Lähteenmäki ${ }^{2}$

1 Tuorla Observatory, Väisäläntie 20, FIN-21500 Piikkiö, Finland

2 Metsähovi Radio Observatory, Helsinki University of Technology, FIN-02540 Kylmälä, Finland

Received March 30; accepted November 26, 1999

\begin{abstract}
We present results of optical monitoring of quasars and blazars obtained at Tuorla Observatory between September 1995 and May 1997. The aim of our monitoring is to provide well sampled optical light curves for quasar and blazar studies. During the observing period the $V$-band brightness of S2 0109+224, 3C 66A, and ON 231 (W Comae) reached some of the brightest values ever measured for these objects. A strong outburst was observed in Mrk 421 and the large outburst, which was predicted earlier, in OJ 287 was confirmed. CTA 102 had over one magnitude outburst which happened in a timescale of a few days. For OI 090.4 we measured one of the faintest $V$-band brightness values ever.
\end{abstract}

Key words: galaxies: active — BL Lacertae objects: general - quasars: general

\section{Introduction}

Blazars show flux variability from radio to $\gamma$-ray energies. Variability timescales are generally shorter at higher energies. Some of the flux variations in different energy regions might have a common origin, for example, simultaneous multifrequency observations have indicated correlations between $\gamma$ - and radio emission (Teräsranta \& Valtaoja 1996) and between optical and $\gamma$-ray emission (Wagner 1996; Bloom et al. 1997). Compton Gamma Ray Observatory (CGRO) discovered in the early 1990's that radio-loud blazars (BL Lacs and HPQs) emit $\gamma$-rays at $\mathrm{MeV}$ - and $\mathrm{GeV}$-energies. Some of them have shown

Send offprint requests to: S. Katajainen,

e-mail: sekataja@astro.utu.fi

* Tables 2 and 3 are only available in electronic form at http://www.edpsciences.org remarkable flux variability at $\mathrm{GeV}$-energies on timescales of a few days. Intensive monitoring will provide information on their variability and will help to study emission processes.

Due to inverse Compton-scattering, the optical photons in the beamed relativistic jet might be scattered to frequencies which are $10^{6}$ times higher than the original frequency. The new frequency should then be in the $\gamma$-ray range. Optical monitoring simultaneous with the $\gamma$-ray satellite pointings gives an opportunity to test how these optical variations correlate with variations in the $\gamma$-ray range.

The optical monitoring of blazars at Tuorla Observatory (University of Turku) started 20 years ago (Sillanpää et al. 1988a, 1991), being one of the oldest quasar monitoring programs in Europe still in progress.

\section{Observations and data-reductions}

The observations presented here were made by using the 1.03 metre Dall-Kirkham-type telescope (f/8.45) of Tuorla Observatory with a SBIG ST-8 CCD-camera and a standard $V$-band filter. A small number of observations were also taken on Roque de los Muchachos (La Palma, Canary Islands) at the 2.56 metre Nordic Optical Telescope (NOT) with standard $B-, V$-, and $R$-filters. Corrections for dark-current effects, additive effects (bias) and multiplicative effects (flatfield) were applied. Due to the relatively small $\left(5^{\prime} 34^{\prime \prime} \times 3^{\prime} 42^{\prime \prime}\right)$ field of view of the 1.03 metre telescope separate frames of comparison stars were exposed for Mrk 421 and 4C 29.45. For the other objects normal differential photometry methods were applied. Exposure times with the Tuorla 1.03 meter telescope were between 60 and 240 seconds for objects brighter than 16 mag. For fainter objects, we have 
combined several exposures to achieve a sufficiently high signal to noise ratio.

Photometric measurements were made with the normal IRAF-routines and applying differential photometry. The error, $\sigma_{\text {total }}$, associated with each measurement was assumed to consist of two components: $\sigma_{\text {total }}=$ $\sqrt{\sigma_{\text {photon }}{ }^{2}+\sigma_{\text {cal }}{ }^{2}}$. The first component, $\sigma_{\text {photon, consists }}$ of the photon noise of the object, the error in sky measurement, and the background photon noise in the measurement aperture, summed in square. The second component, $\sigma_{\text {cal }}$, was obtained from the standard error of the mean of the magnitude difference between the object and its comparison stars. Typical total errors were between 0.03 and 0.06 mag.

The objects in our sample have been chosen by the following selection criteria:

1) radio-loud, $\gamma$-bright blazar (Fichtel et al. 1994; von Montigny et al. 1995.; Thompson et al. 1995),

2 ) optically bright enough $(V \leq 18 \mathrm{mag})$ to be observed with a telescope of one metre aperture.

Several objects in our sample were targets of EGRETpointings during the years 1995, 1996, and 1997. Some blazars which have not been detected in $\gamma$-rays were also selected for our sample so as to compare their variability properties to those of $\gamma$-bright blazars. Some objects, like OJ 287, were monitored as a part of the OJ-94 campaign (Sillanpää et al. 1996a-c). A list of the objects including their names, coordinates (epoch 2000.0), redshift, classification, number of datapoints observed at Tuorla, variation scale in the $V$-band $(\Delta V)$, detection in the $\gamma$-region (Fichtel et al. 1994; von Montigny et al. 1995; Thompson et al. 1995; Catanese et al. 1997), and comparison stars with their references is given in Table 1.

\section{Results}

A short presentation of each object with some references to earlier photometric studies is given. Numerical data (Tables 2 and 3) are available only in electronic form at the CDS via anonymous ftp. Table 2 includes the data observed at Tuorla with the 1.03 metre telescope ( $V$-band). Table 3 includes data ( $B$-, $V$-, and $R$-bands) observed at the Nordic Optical Telescope (NOT) on La Palma. Due to the modest amount of the $B$ - and $R$-band data, a detailed analysis for that data was not possible. A few $V$-band datapoints observed at the NOT are included in the light curves. Otherwise the light curves present the $V$-band data that was obtained at Tuorla. The notation $\overline{\mathrm{JD}}=\mathrm{JD}-2400000$ is used throughout the text and in the light curves.

For those objects which have been targets of large multifrequency campaigns, detailed analysis and numerical data can be found from the following articles: Takalo et al. (1998) (AO 0235+164); Raiteri et al. (1999) (S4 0954+658); Tosti et al. (1998a) (Mrk 421); Tosti et al. (1998b) (ON 231).

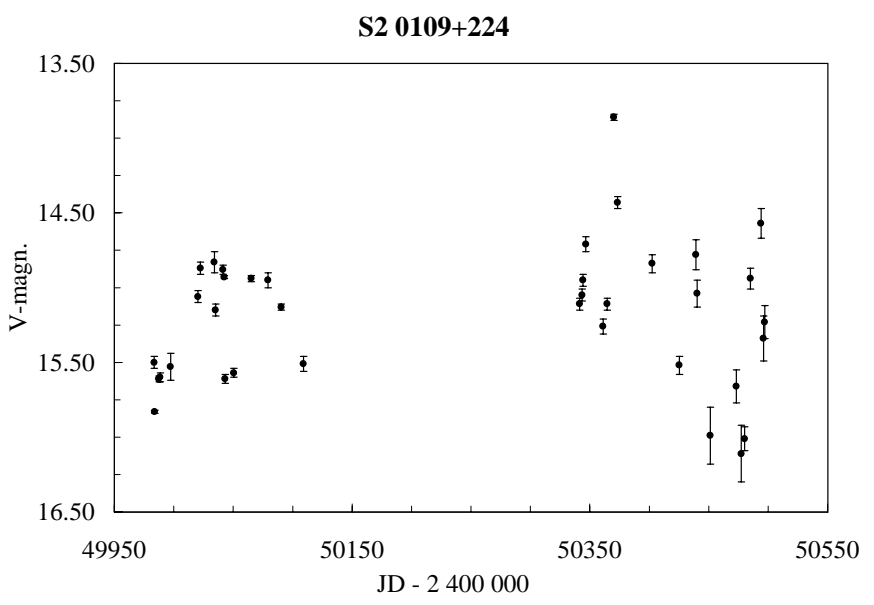

Fig. 1. Light curve of S2 $0109+224$

\section{1. $520109+224$}

S2 0109+224 (PKS 0109+224) was first identified optically by Owen \& Mufson (1977). The largest brightness variations in the historical light curve occurred in 1943: a drop of 3.07 mag during one year (Pica 1977). Typical for S2 0109+224 have been one mag flux variations on a timescale of $1-2$ years. The average brightness in the $B$ band has been $16.41 \mathrm{mag}$, and flickering of $0.6-0.7 \mathrm{mag}$ has been observed by Pica et al. (1988).

The brightness in the $V$-band varied during our observations by between 13.86 and 15.83 mag. Rapid flux variations were seen frequently (Fig. 1). S2 0109+224 was exceptionally bright during 1996-1997 (JD 50340-50500) compared to the earlier monitoring campaigns (Pica et al. 1988; Sillanpää et al. 1988a; Takalo 1991; Xie et al. 1994; Villata et al. 1997; Raiteri et al. 1998). The maximum, 13.86 ( $\overline{\mathrm{JD}} 50370)$, is one of the brightest $V$-band values measured for this object.

\section{2. $3 C 66 A$}

3C 66A (PKS 0219+428) was first identified optically by Wills \& Wills (1974). Apparently it is located near a large cluster of faint galaxies and near the radio galaxy $3 \mathrm{C} 66 \mathrm{~B}$. For earlier optical observations see Takalo et al. (1996 and references therein).

The light curve of $3 \mathrm{C} 66 \mathrm{~A}$ (Fig. 2) shows continuous $0.2-0.3 \mathrm{mag}$ variations. One of the brightest $V$-band mag ever for 3C 66A, 13.61, was measured on November 1995

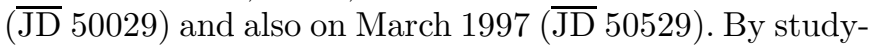
ing the light curve (Fig. 2) it seems that the local minima occur about every $60-70$ days. Stronger evidence for periodicity of about 65 days can be found in the analysis made by Lainela et al. (1999). 
Table 1. Objects in Tuorla quasar monitoring campaign

\begin{tabular}{|c|c|c|c|c|c|c|c|c|}
\hline Name & R.A.(2000) & Decl.(2000) & $z$ & class. & $N$ obs. & $\Delta V$ & $\gamma$-detec. & comp. stars ref. \\
\hline S2 $0109+224$ & 011205.8 & +224439 & unknown & BL Lac & 38 & 1.97 & No & 8 \\
\hline $3 \mathrm{C} 66 \mathrm{~A}$ & 022239.6 & +430208 & 0.444 & BL Lac & 74 & 1.16 & Yes & 4,5 \\
\hline AO $0235+164$ & 023838.9 & +163700 & 0.94 & BL Lac & 7 & $*$ & Yes & 10 \\
\hline OF 038 & 042446.7 & +003607 & unknown & BL Lac & 16 & 1.10 & No & 8 \\
\hline S5 $0716+714$ & 072153.3 & +712036 & unknown & BL Lac & 108 & 1.58 & Yes & 5,11 \\
\hline PKS $0735+178$ & 073807.4 & +174219 & 0.424 & BL Lac & 41 & 1.68 & Yes & 10 \\
\hline PKS $0736+107$ & 073918.0 & +013704 & 0.191 & HPQ & 19 & 0.59 & No & 10 \\
\hline OI 090.4 & 075706.7 & +095635 & 0.660 & BL Lac & 38 & 1.03 & No & 8 \\
\hline OJ 287 & 085448.8 & +200630 & 0.306 & BL Lac & 120 & 1.68 & No & 5 \\
\hline $\mathrm{S} 40954+658$ & 095847.2 & +653354 & 0.367 & BL Lac & 35 & $*$ & Yes & 9 \\
\hline Mrk 421 & 110427.2 & +381232 & 0.031 & BL Lac & 47 & $*$ & Yes & 14 \\
\hline OM 280 & 115019.2 & +241754 & 0.200 & BL Lac & 26 & 0.63 & No & 5 \\
\hline $4 \mathrm{C} 29.45$ & 115931.9 & +291445 & 0.729 & HPQ & 11 & 1.60 & Yes & 10 \\
\hline ON 325 & 121752.0 & +300701 & 0.237 & BL Lac & 37 & 0.83 & No & 5 \\
\hline ON 231 & 122131.7 & +281358 & 0.102 & BL Lac & 73 & $*$ & Yes & 6 \\
\hline $3 \mathrm{C} 273$ & 122906.7 & +022908 & 0.158 & LPQ & 54 & 0.30 & Yes & 10 \\
\hline 3C 279 & 125611.1 & -054721 & 0.538 & HPQ & 45 & 1.5 & Yes & 12 \\
\hline PKS $1424+240$ & 142700.5 & +234800 & unknown & BL Lac & 25 & 0.21 & No & 5 \\
\hline 4C 38.41 & 163515.5 & +380805 & 1.814 & LPQ & 5 & 1.61 & Yes & 13 \\
\hline $3 \mathrm{C} 345$ & 164258.8 & +394837 & 0.595 & HPQ & 40 & 1.03 & No & 10 \\
\hline OT 546 & 172818.6 & +501311 & 0.055 & BL Lac & 25 & 0.59 & No & 4 \\
\hline 3C 371 & 180650.7 & +694928 & 0.050 & BL Lac & 26 & 0.79 & No & 7 \\
\hline BL Lacertae & 220243.3 & +421639 & 0.069 & BL Lac & 52 & 1.34 & Yes & 3 \\
\hline 4C 31.36 & 220314.5 & +314542 & 0.297 & LPQ & 20 & 0.63 & No & 12 \\
\hline CTA 102 & 223236.4 & +114351 & 1.037 & $\mathrm{HPQ}$ & 9 & 2.01 & Yes & 2 \\
\hline $3 \mathrm{C} 454.3$ & 225357.7 & +160853 & 0.859 & HPQ & 25 & 0.78 & Yes & 1 \\
\hline OY 091 & $2257 \quad 17.3$ & +074312 & 0.190 & BL Lac & 15 & 0.52 & No & 4 \\
\hline
\end{tabular}

(1) Angione (1971) (2) Balonek, http://quasar.colgate.edu/ tbalonek/optical/CTA102compstars.gif (3) Bertaud et al. (1969)

(4) Crane (1977) (5) Fiorucci et al. (1996) (6) Fiorucci et al. (1998) (7) McGimsey et al. (1975) (8) Miller et al. (1983)

(9) Raiteri et al. (1999) (10) Smith et al. (1985) (11) Takalo et al. (1994) (12) Tritton et al. (1973) (13) Villata et al. (1997).

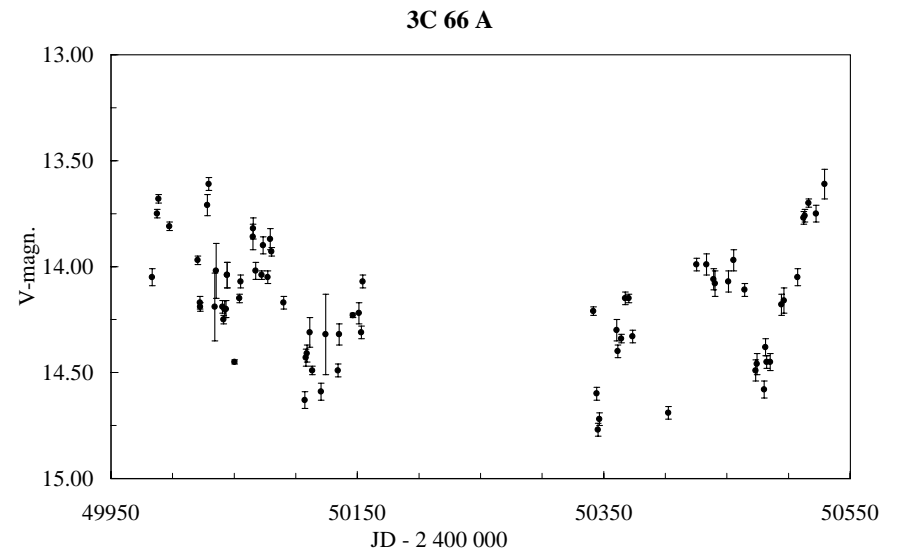

Fig. 2. Light curve of $3 \mathrm{C} 66 \mathrm{~A}$

\section{3. $A O 0235+164$}

AO $0235+164$ (OD 160) is one of the most variable BL Lac objects. The few datapoints observed at Tuorla, when AO $0235+164$ was in its faint state $(V \geq 18)$, are published with the results of a multifrequency campaign by Takalo et al. (1998).

\subsection{OF 038}

OF 038 (PKS 0422+004) was first classified as a BL Lac object by Kinman (1976a, 1976b). Eachus \& Liller (1975) found from Harvard College Observatory's Plate archive that the total amplitude variation was 3 mag. Burbidge \& Hewitt (1989) observed the faintest known $V$-band magnitude, 17.0. The average $B$-band brightness in Rosemary Hill Observatory's long term monitoring (Pica et al. 1988) was 16.05 mag. Fast 0.3 mag flux variations have been typical for this object during observations by Xie et al. (1994 and references therein). The brightest $V$-band measurement has been made by Massaro et al. (1996), $V=13.65$ on November 1994.

Our OF 038 light curve (Fig. 3) showed a fading of almost one mag during two months in 1996 (JD 50090 and $\overline{\mathrm{JD}} 50134)$. During the next observing season (19961997 ) the flux of OF 038 rose continuously and reached 


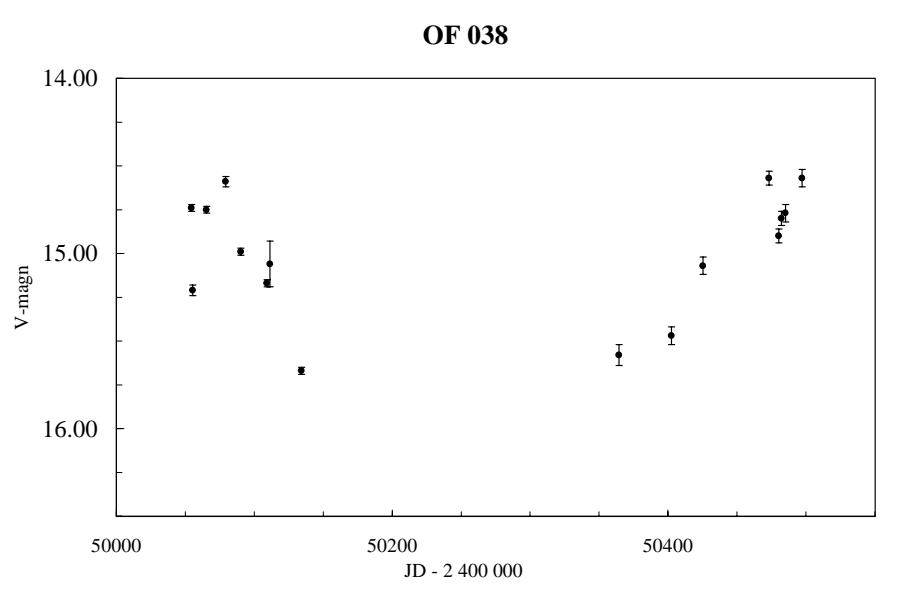

Fig. 3. Light curve of OF 038

14.57 mag twice ( $\overline{\mathrm{JD}} 50473$ and $\overline{\mathrm{JD}} 50497)$. The faintest $V$-band measurement was $15.67 \mathrm{mag}$ ( $\overline{\mathrm{JD}} 50134)$.

\section{5. $S 50716+714$}

S5 $0716+714$ is a radio-loud flat-spectrum source discovered in the Bonn-NRAO radio survey (Kühr et al. 1981, 1987). It was classified as a BL Lac object by Biermann et al. (1981), who measured two photographic magnitudes (13.2 and 15.5) from the POSS plates. During the 1990's this source has been intensively studied at all frequencies. Quirrenbach et al. (1991) noticed correlated radio and optical changes on timescales of a few days. Wagner et al. (1996) reported rapid variations over the whole electromagnetic spectrum. S5 0716+714 is a strong EGRETsource and its $\gamma$-flux has shown considerable variability (Lin et al. 1995).

The light curve of S5 $0716+714$ (Fig. 4) is one of the best sampled among our objects. Our $V$-band light curve shows a "sawtooth"-pattern. It is interesting to notice in our data that the maximum points in the light curve (Fig. 4) seem to occur with a period of $60-70$ days. These values, 14.07, 14.12, 14.13 and 14.16, during 1995-1996 observations, have only $0.09 \mathrm{mag}$ deviation. The average brightness of S5 $0716+714$ was rising during 1996-1997. The minimum value of our light curve is 15.15

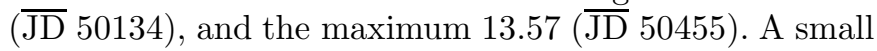
part of observations were simultaneous with one EGRET pointing of S5 $0716+714$ (marked with the box in Fig. 4).

\section{6. $P K S 0735+178$}

PKS $0735+178$ (OI 158) was first optically identified by Blake (1970) as a 16th mag blue stellar-like object. The optical brightness has varied between 13.90 and 17.72 mag in the B-band (Pollock et al. 1979; Xie et al. 1994 and references therein).

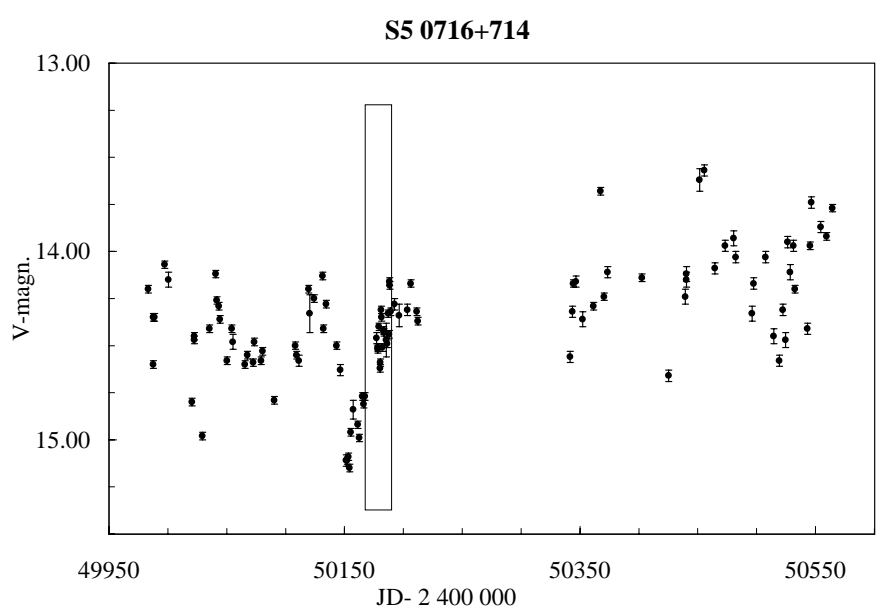

Fig. 4. Light curve of S5 $0716+714$. The box marks a simultaneous EGRET-pointing

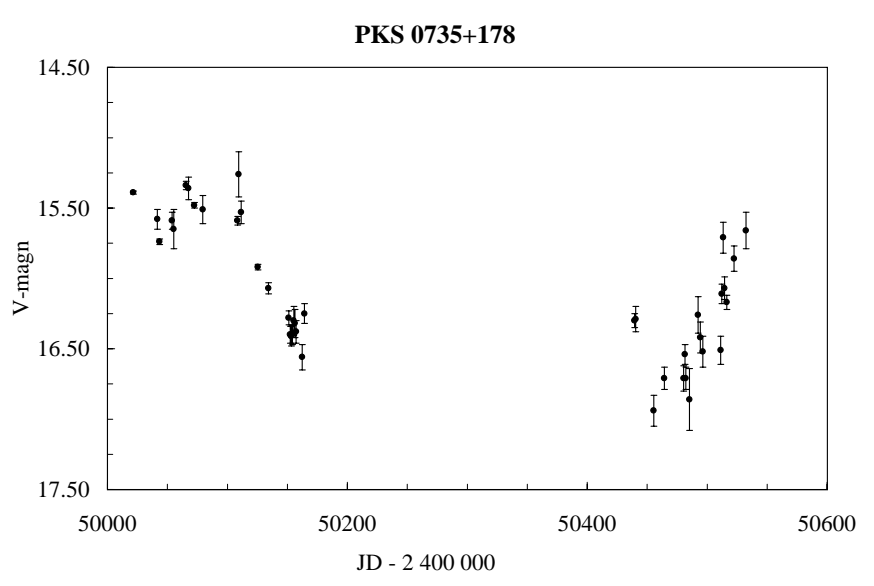

Fig. 5. Light curve of PKS $0735+178$

The brightness of PKS 0735+178 was near $~ 15.5$ during winter 1996, until on February 1996 the object started to fade. The object reached 15.5 level again in late March 1997. The maximum $V$-band brightness during our observations was 15.26 ( $\overline{\mathrm{JD}} 50109)$ and the minimum 16.94 ( $\overline{\mathrm{JD}} 50455)$ (Fig. 5).

\section{7. $P K S 0736+107$}

PKS 0736+107 (OI 061) is classified as a high polarization quasar, HPQ. McGimsey et al. (1975) observed one mag optical variations in PKS 0736+107. Pica et al. (1980, 1988) reported $0.7 \mathrm{mag}$ fades and flares and found from photographic plates that historically the brightness had been between mag 15.45 and 16.80 in the $B$-band.

During our observations between 1995 and 1997 the brightness varied between $16.25(\overline{\mathrm{JD}} 50494)$ and 16.84 (JD 50512) mag (Fig. 6). 


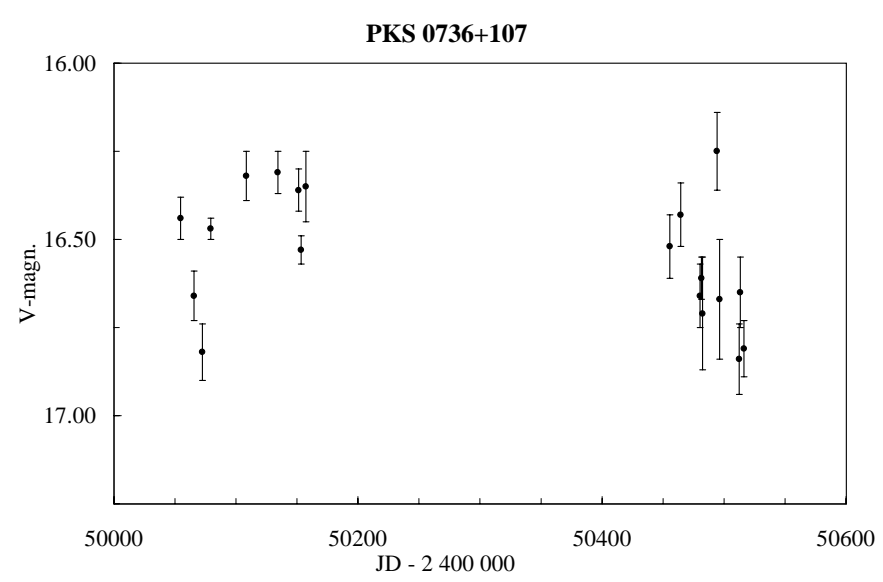

Fig. 6. Light curve of PKS $0736+107$

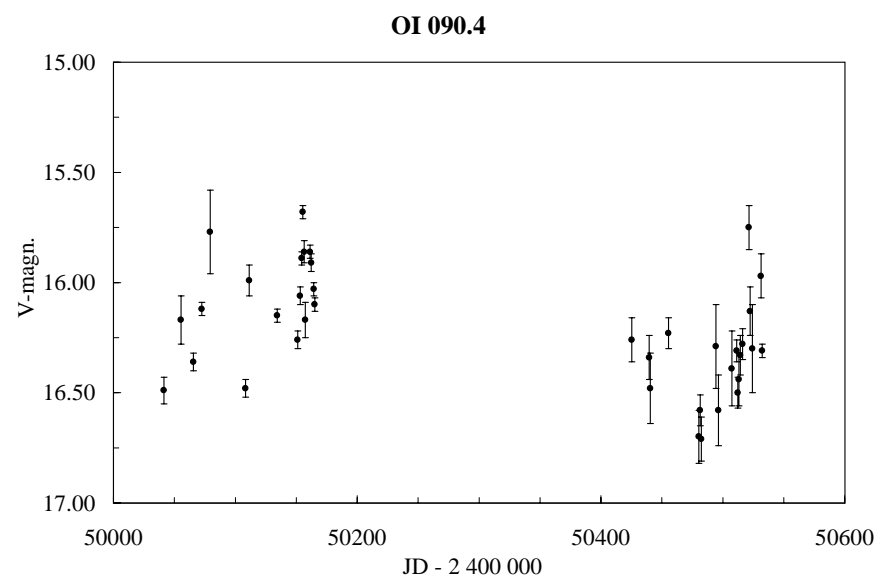

Fig. 7. Light curve of OI 090.4

\subsection{OI 090.4}

OI 090.4 (PKS 0754+110) was identified as a BL Lacobject by Tapia et al. (1977). Its historical light curve has been studied by Baumert (1980), who found 0.8 mag variations on timescales of a few days and a variability of 2 mag in a few years. Pica et al. (1988) observed 0.8 magnitude ( $B$-band) changes on a 2 year timescale. Zekl et al. (1981) reported on $2 \mathrm{mag}$ fluctuations in the $B$-band. Sillanpää et al. (1991) observed $0.9 \mathrm{mag}$ variations in the $V$-band. Smith et al. (1987) have measured $V=16.31$, which has been so far the faintest $V$-band value for this object.

During winter 1996 and winter 1997 the average brightness was between 16 and 16.5 (Fig. 7). The minimum, 16.71, ( $\overline{\mathrm{JD}} 50482$ ) was observed on February 1997 and is one the faintest $V$-band measurement for this object (see references above) ever. The brightest $V$-band value measured was 15.68 ( $\overline{\mathrm{JD}} 50155)$.

\subsection{OJ 287}

OJ 287 (PKS 0851+202) is one of the most studied extragalactic objects from radio frequencies to $\mathrm{X}$-ray

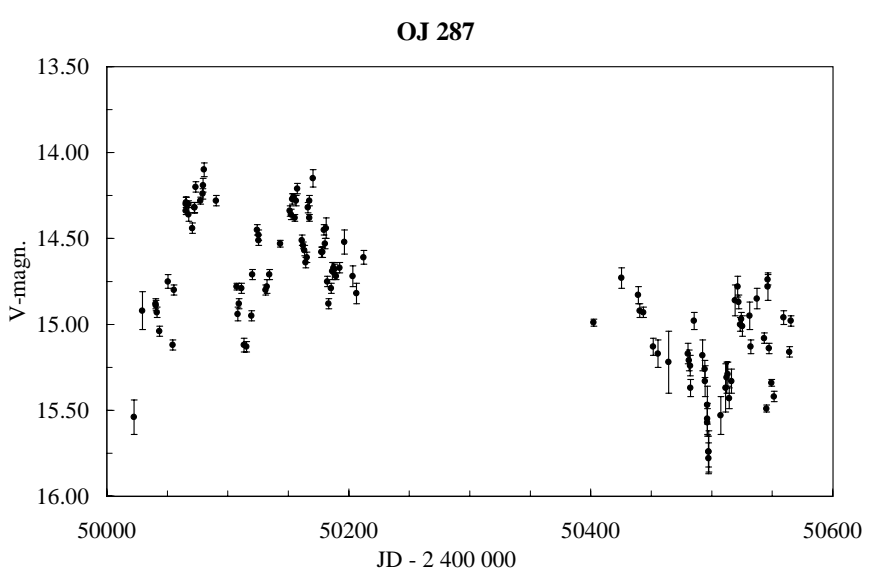

Fig. 8. Light curve of OJ 287

frequencies. It was first optically identified by Blake (1970) as a $14.5 \quad B$-magnitude stellar-like object. OJ 287 has been the target of many optical observations since the 1970's, including long-term optical monitoring programs, such as the one at Rosemary Hill Observatory (Webb et al. 1988), where over 5 mag $B$-band variations have been measured. Craine (1977) reported that the brightness of OJ 287 in the $V$-band has varied between 12.7 and 15.6. For a complete historical overview of this object see Takalo (1994). Sillanpää et al. (1988a) measured values from 12.64 to $15.50 \mathrm{mag}$ in the $V$-band between 1982 and 1984. During those observations this source was in an outburst state. Previous large outbursts had happened in 1911, 1937, 1947, 1954 and 1971/1972. Analysing this historical light curve Sillanpää et al. (1988b) demonstrated that the outbursts have a period of $\sim 12$ years, and explained this behaviour by a supermassive binary black hole model. They also predicted that OJ 287 should be in an outburst state again during years 1994 and 1995. This was confirmed by the observations in the OJ-94 project (see Sillanpää et al. 1996a-c).

The OJ 287 observations presented here cover the second peak (1.2 years after the first peak) of the predicted outburst (Sillanpää et al. 1996a-c). The $V$ band brightness reached 14.10 on 28 December 1995 (JD 50080) (Fig. 8), and 14.15 was reached on 27 March 1996 ( $\overline{\mathrm{JD}}$ 50170). During the next observing season, winter 1997 OJ 287 became gradually fainter, and the brightness dropped rapidly $0.8 \mathrm{mag}$ in 12 days on February 1997, when a minimum, 15.78 ( $\overline{\mathrm{JD}} 50497)$, was reached.

\subsection{0. $S 40954+658$}

The radio source S4 0954+658 (Pauliny-Toth et al. 1978) has been monitored quite intensively by Wagner et al. (1993), Heidt (1993) and Raiteri et al. (1999). It has been classified as an intraday variable (IDV) in the radio and optical regions. 


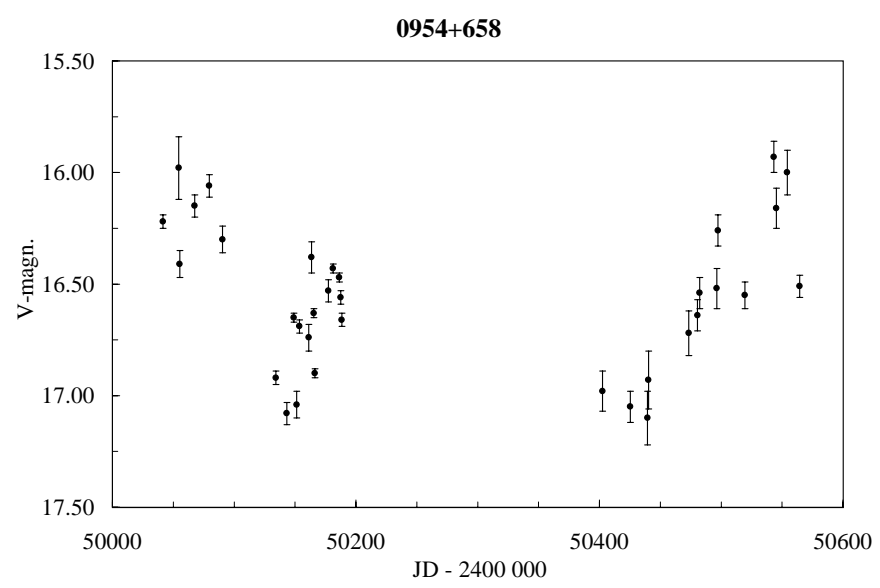

Fig. 9. Light curve of S4 0954+658

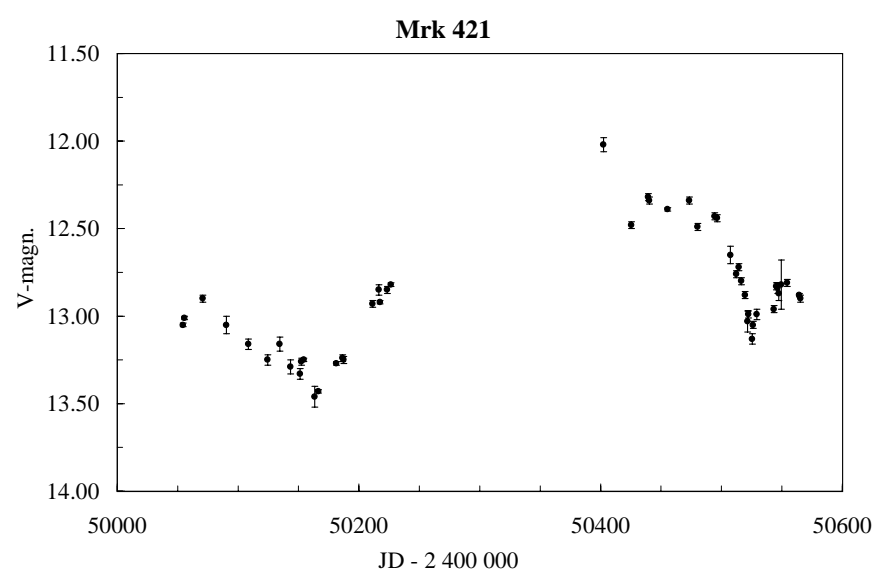

Fig. 10. Light curve of Mrk 421

Numerical data and a detailed analysis of our S4 $0954+658$ observations is published by Raiteri et al. (1999), and only the light curve is presented here (Fig. 9).

\subsection{Mrk 421}

Mrk 421 (Markarian \& Lipovetsky 1972) has shown strong and rapid TeV-outbursts several times (e.g. Schubnell et al. 1996; Petry et al. 1996). Our data and analysis of the optical behaviour of Mrk 421 are presented by Tosti et al. (1998a) and hence only the light curve is shown (Fig. 10).

\subsection{2. $O M 280$}

OM 280 (QSO $1147+245)$ is classified as a BL Lac object (Angel \& Stockmann 1980). The amplitude of the $B$-band variability has been about $0.8 \mathrm{mag}$, with the average brightness 16.6 during 1981-1986 (Pica et al. 1988). Zekl et al. (1981) reported $0.4 \mathrm{mag}$ variations in the $B$ band. Moles et al. (1985) measured 16.02 as the average

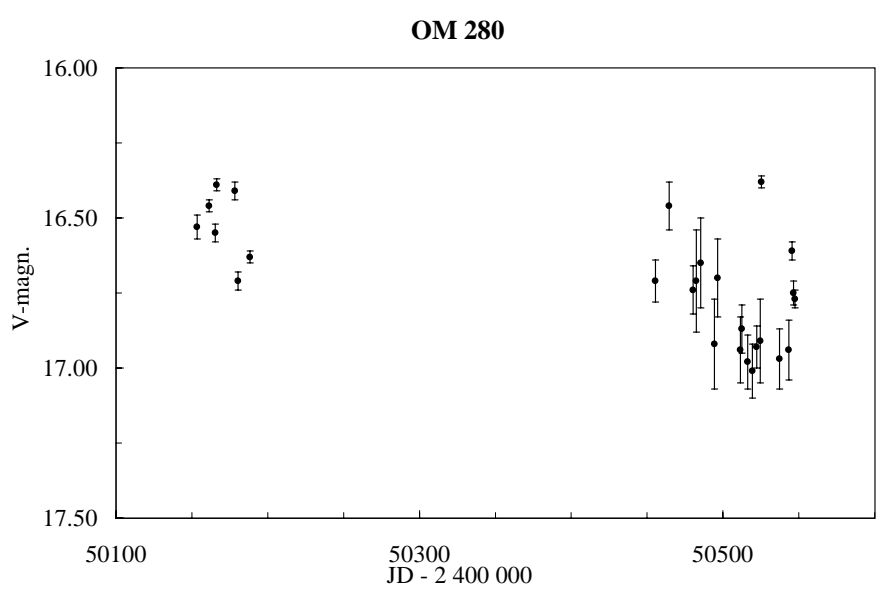

Fig. 11. Light curve of OM 280

brightness in the $V$-band. During the recent years Fiorucci \& Tosti (1996b) reported that the $V$-band brightness was 16.15 as an average.

Our data indicate that the brightness in the $V$-band was near 16.5 mag during winter 1996 and between 16.5 and 17.0 during winter 1997 (Fig. 11). The brightest $V$-band value was 16.38 ( $\overline{\mathrm{JD}} 50525)$ and the faintest 17.01 (JD 50519) mag.

\subsection{3. $4 C 29.45$}

4C 29.45 (PKS 1156+295) belongs among to the most variable OVV-quasars. Studies of photographic plates have shown that the average brightness of this object has been $\sim 16$ mag in the $B$-band. On March 1981 it had an outburst of $5 \mathrm{mag}$, starting from $B=18$ and rising up to 13.15 (Wills et al. 1983). In 1985 a 2.5 mag outburst occurred (Miller 1985). 4C 29.45 is a strong GeV-source (Thompson et al. 1995; Webb et al. 1995).

The $V$-band brightness of $4 \mathrm{C} 29.45$ varied between $16.12(\overline{\mathrm{JD}} 50125)$ and 16.68 ( $\overline{\mathrm{JD}} 50134$ and $\overline{\mathrm{JD}}$ 50143) during 1995-1996. In winter 1997 we observed only two datapoints (15.45) ( $\overline{\mathrm{JD}}$ 50455) and 17.05 (JD $50480 \mathrm{mag}$ ) for this object. Due to small number of data (Table 2) no light curve is shown.

\subsection{ON 325}

ON 325 (PKS 1215+303) was identified as a quasar by Browne (1971), and confirmed by Veron \& Veron (1973). It was later classified as a BL Lac object. Veron \& Veron (1975) reported $2.7 \mathrm{mag}(14.3-17.0)$ changes in the $B$-band. Observations made at Rosemary Hill Observatory during 16 years (Pica et al. 1988) showed over 2 mag $B$-band variations with an average brightness of 15.7. At least in 1974, 1977, 1980, 1985, and 1986, over one magnitude outbursts have occured. Zekl et al. (1981) reported that the brightness of this source has historically been between 13.7 and $17.0 \mathrm{mag}$ in the $B$-band. 


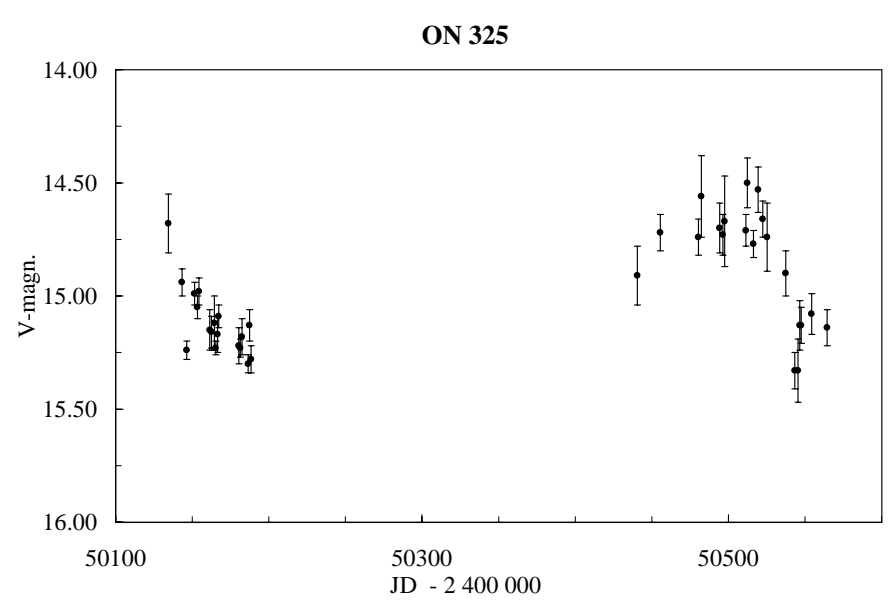

Fig. 12. Light curve of ON 325

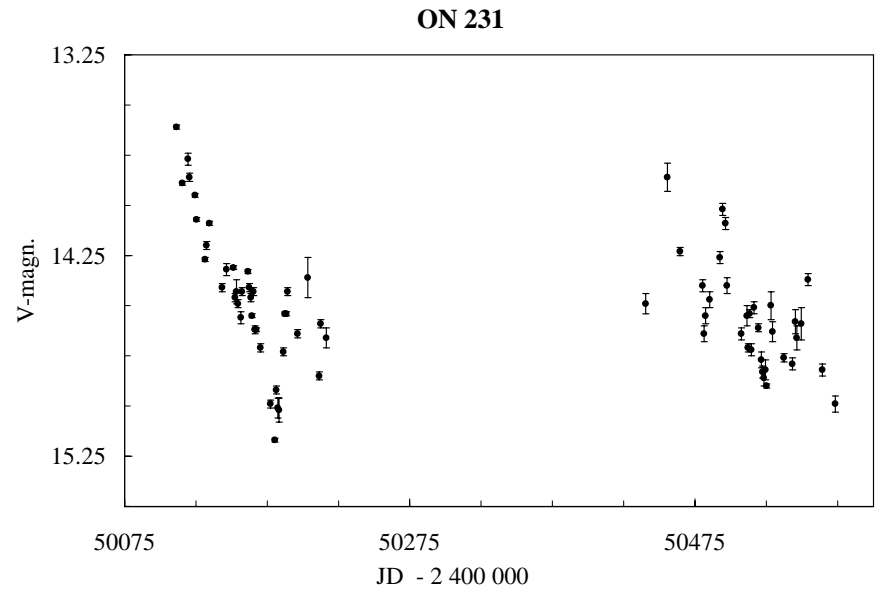

Fig. 13. Light curve of ON 231

The data from the first observing season (1995-1996) show (Fig. 12) a smooth decrease in brightness from 14.7 to $15.3 \mathrm{mag}$. Observations during winter 1997 show a maximum point in the light curve, $14.50 \mathrm{mag}(\overline{\mathrm{JD}} 50512)$ and a fade from that to 15.33 (JD 50543), the minimum in our ON 325 observations.

\subsection{ON 231}

ON 231 (QSO 1219+285) was identified by Browne (1971) and Biraud (1971) as a stellar like object, which was the same as the variable star W Comae. Historical light curve has been studied by Liu et al. (1995).

Our ON 231 data are published and analysed by Tosti et al. (1998b), so only the light curve is presented (Fig. 13).

\subsection{C 273}

3C 273 (PKS 1226+02) was the first QSO discovered. It is classified as a low polarization quasar (LPQ). The optical variations are usually relatively small, even the

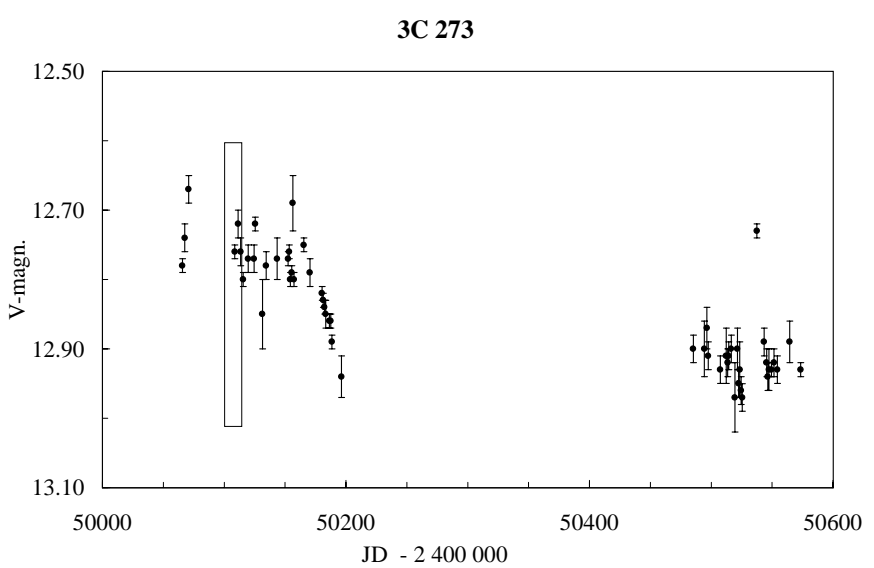

Fig. 14. Light curve of $3 \mathrm{C} 273$ The box marks a simultaneous EGRET-pointing

total brightness variation amplitude has been less than one magnitude (Pica et al. 1988; Sillanpää et al. 1988a). In 1983 brightness reached $12.17 \mathrm{mag}$ in the $V$-band (Sillanpää et al. 1988a). Studies by Fiorucci \& Tosti (1996b) and Villata et al. (1997) did not show any large optical variations.

During our observations on December 1995 3C 273 was brightening rapidly (Fig. 14) (between $\overline{\mathrm{JD}} 50065$ and $\overline{\mathrm{JD}}$ 50070). On January 1996 the object did not show a continuation of this trend. Part of the observations on January and February 1996 were simultaneous with an EGRET-pointing (marked with a box in Fig. 14). In early April 1996 ( $\overline{\mathrm{JD}}$ 50157) 3C 273 started to fade from mag 12.8 towards mag 13 . The shape of our $V$-band light curve observed during winter 1996 is also in agreement with the $R$-band variations observed by Raiteri et al. (1998) during the same period. Observations during winter 1997 did not show large flares, the brightness was near 12.9 mag. The maximum $V$-band brightness during years 1995 and 1997 was 12.67 ( $\overline{\mathrm{JD}} 50070)$ and the minimum $12.97(\overline{\mathrm{JD}} 50519$ and $\overline{\mathrm{JD}} 50525)$ mag.

\subsection{7. $3 C 279$}

3C 279 (PKS 1253-055) is a high polarization quasar (HPQ) and one of the most violent variable sources in the sky, showing 8 mag variations in its historical light curve (Webb et al. 1990). 3C 279 had large outbursts at least during 1936, 1938, 1987, and 1992. Normally the variation amplitude in the optical region has been about 2 mag. This object is one of the strongest EGRET $\gamma$-ray sources (Thompson et al. 1995; Wehrle et al. 1998).

Several 3C 279 datapoints are simultaneous with an EGRET pointing (marked with a box in Fig. 15). On January and February 1996 ( $\overline{\mathrm{JD}} 50100)$ 3C 279 was brightening rapidly in optical. The EGRET pointing on February 1996 showed also a fast rise in the GeV $\gamma$-ray flux. The results of the multifrequency campaign 


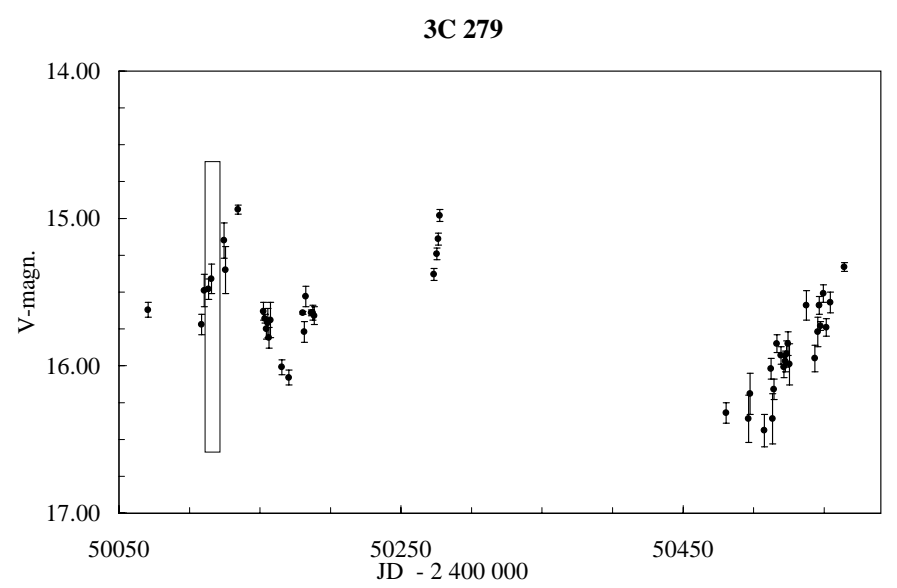

Fig. 15. Light curve of $3 \mathrm{C} 279$ The box marks a simultaneous EGRET-pointing

during this particular $\gamma$-flare are studied by Wehrle et al. (1998). The optical brightening was over on March and the object faded to $V$-magnitude 16 . The four datapoints in the light curve (Fig. 15) between $\overline{\mathrm{JD}} 50273$ and JD 50277 have been observed at the NOT in summer 1996. They show that 3C 279 brightened $0.4 \mathrm{mag}$ in four days. Observations during winter 1997 showed that 3C 279 was fainter than mag 16 . The brightness rose in spring 1997, reaching mag 15.2. The maximum ( $V$-band) brightness during our observations was 14.94 ( $\overline{\mathrm{JD}} 50134)$ and the minimum ( $\overline{\mathrm{JD}} 50507) 16.44$.

\subsection{PKS $1424+240$}

PKS $1424+240$ is a radio source which was identified as a blazar by Impey \& Tapia (1988). Originally it was classified as a white dwarf in the Palomar-Green Survey. Studies by Fleming et al. (1993) from Rosat All Sky Survey (RASS) showed PG $1424+240$ to be a BL Lac object. Fiorucci \& Tosti (1996b) reported that the brightness in the $R$-band was about 14.3 mag.

During our PKS $1424+240$ observations the average $V$-band magnitude was $\sim 14.5$ (Fig. 16). The range of variation was quite small, which is in agreement with the earlier observations of Fiorucci \& Tosti (1996b). The minimum value for the brightness was $14.63 \overline{\mathrm{JD}} 50186)$ and the maximum 14.42 ( $\overline{\mathrm{JD}}$ 50573).

\subsection{9. $4 C 38.41$}

4C 38.41 (QSO 1633+38) is classified as a quasar in some references. Mattox et al. (1993) classified it as an OVV quasar. It faded over $3 \mathrm{mag}$, according to the observations of Barbieri et al. (1977), between years 1969 and 1976. It is a strong EGRET source (Mattox et al. 1993; Fichtel et al. 1994; Thompson et al. 1995).

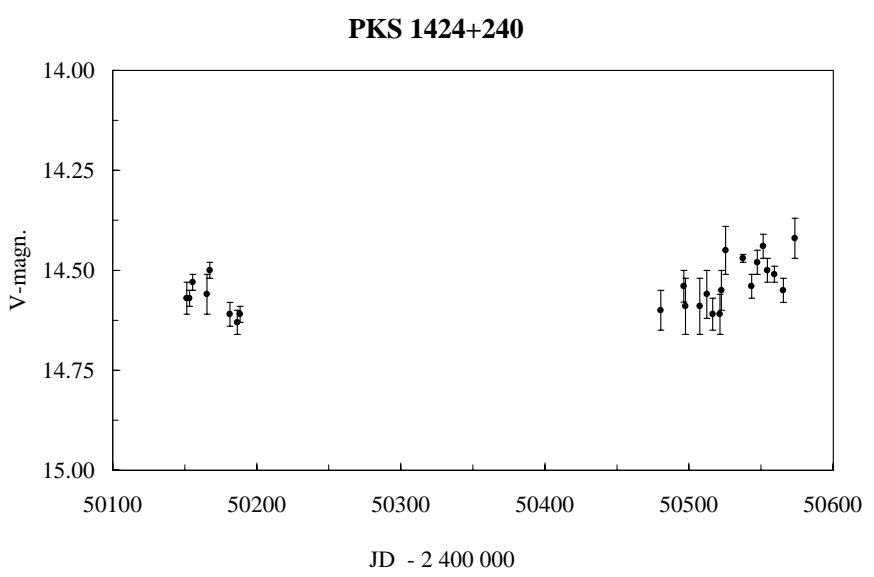

Fig. 16. Light curve of PKS $1424+240$

We observed 4C 38.41 several times in Tuorla during 1995-1997. The observations showed that the object was at first in a faint state, $17.55 \mathrm{mag}$ (minimum). By the next winter the brightness had risen to mag 16 (maximum 15.94 at $\overline{\mathrm{JD}}$ 50531), from which it faded to 17.35 ( $\overline{\mathrm{JD}} 50554$ ). Due to the small number of data (Table 2) no light curve is shown.

\subsection{0. $3 C 345$}

3C 345 (S4 1641+399) was classified as an OVV quasar by Penston \& Cannon (1970). Typical for this object have been about 2 mag outbursts, which occur quite frequently. Such large outbursts happened in 1967/1968, 1971/1972, 1982/1983, and 1991/1992 (Schramm et al. 1993). Sillanpää et al. (1988a) measured $V=14.56$ in 1982, while Schramm et al. (1993) observed $V$-band brightness near $18 \mathrm{mag}$ in 1989. In 1991/1992 a large $2.5 \mathrm{mag}$ outburst was observed (Schramm et al. 1993). Kidger \& Takalo (1990) observed the historical minimum $B=18.66$.

During our observations the average brightness of 3C 345 was fainter than mag 17 (Fig. 17). In winter 1996 we observed several $\sim 0.5$ mag brightenings in a timescale of a few days. An EGRET-pointing during our optical observations is marked with a box in Fig. 17. The minimum value for the brightness was 17.77 ( $\overline{\mathrm{JD}} 50152$ ), and the maximum 16.60 ( $\overline{\mathrm{JD}} 50254$, observed at the NOT on June 1996).

\subsection{OT 546}

Strong continuum emission from the nucleus of OT 546 (PKS 1727+502, ZW I 187) was first noticed by Zwicky (1966). Optical variations of this object were reported by Sandage (1967). Angel \& Stockmann (1980) classified it as a BL Lac object. Its historical light curve shows variations between mag 14.8 and 16.9 in the $B$-band (Hall \& 
$3 C 345$

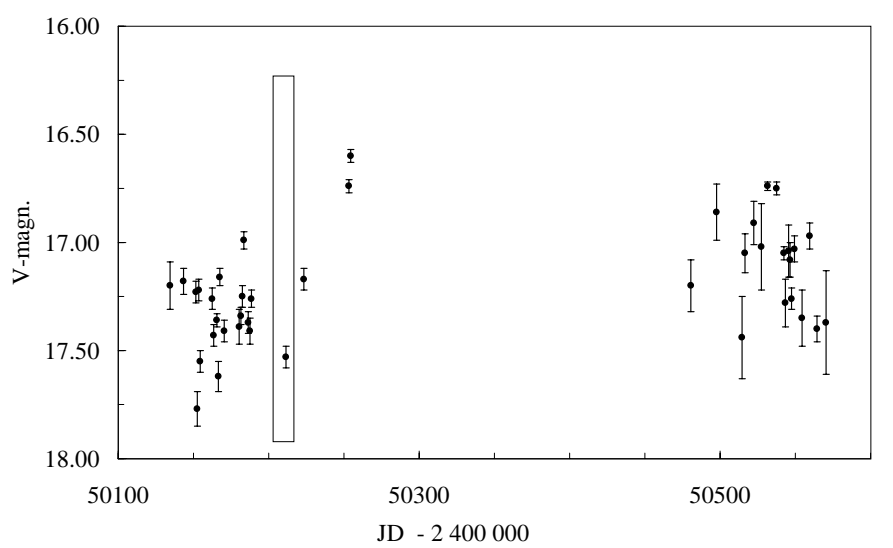

Fig. 17. Light curve of $3 \mathrm{C} 345$ The box marks a simultaneous EGRET-pointing

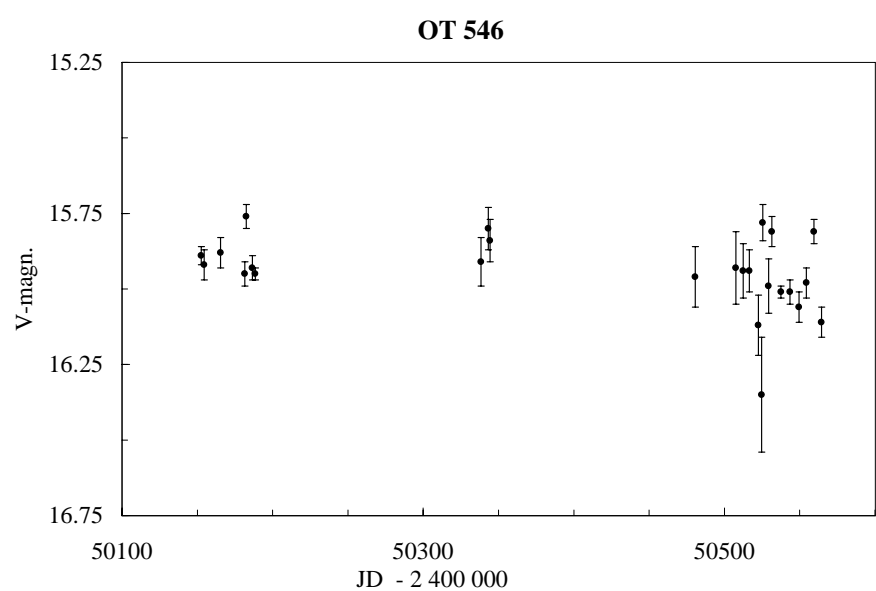

Fig. 18. Light curve of OT 546

Usher 1972). The average brightness in the $V$-band has been near mag 16 (Kinman 1976b). The light curves from Rosemary Hill Observatory observations between years 1975 and 1987 show $0.8 \mathrm{mag}$ variations, with the average $B \sim 16.7$ (Pica et al. 1988).

During our $V$-band observations, the brightness varied between mag 15.76 ( $\overline{\mathrm{JD}} 50182$ ) and 16.35 ( $\overline{\mathrm{JD}} 50524)$ (Fig. 18). The faintest datapoint, 16.35 has, however, fairly large errors $0.19 \mathrm{mag}$. Using the second faintest measurement, 16.12, to calculate the total range of flux variation, the amplitude is only $0.36 \mathrm{mag}$.

\subsection{2. $3 C 371$}

3C $371(\mathrm{~S} 41807+69)$ is a BL Lac object in a large elliptical galaxy whose emission is dominated by the flux from the nucleus. Earlier it was classified as an $\mathrm{N}$ galaxy. Observations by Sandage (1967) showed that 3C 371 was optically variable.

The brightness in the $V$-band was between 14.6 and $15.0 \mathrm{mag}$ in autumn 1996 (Fig. 19). In spring 1997 ( $\overline{\mathrm{JD}} 50520)$ the brightness was rising towards mag 14.3.

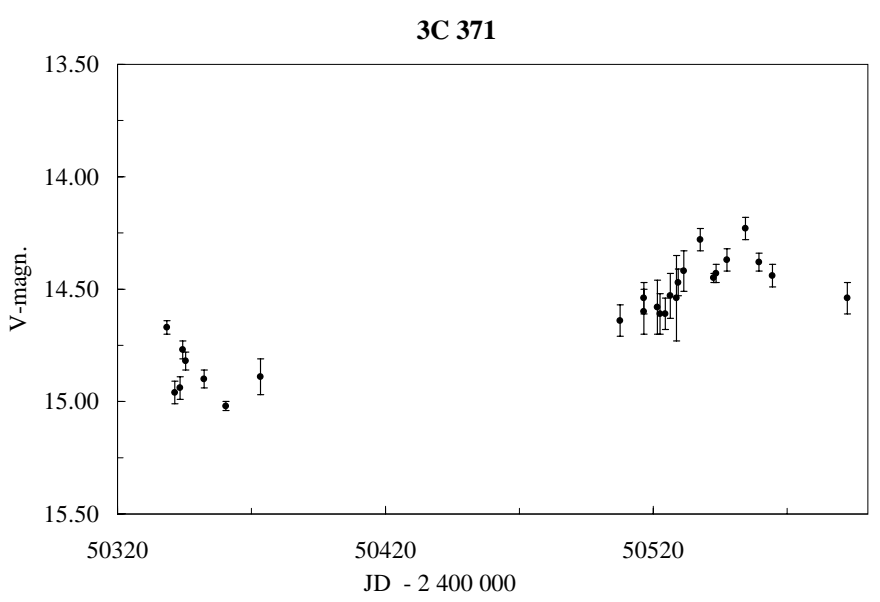

Fig. 19. Light curve of 3C 371

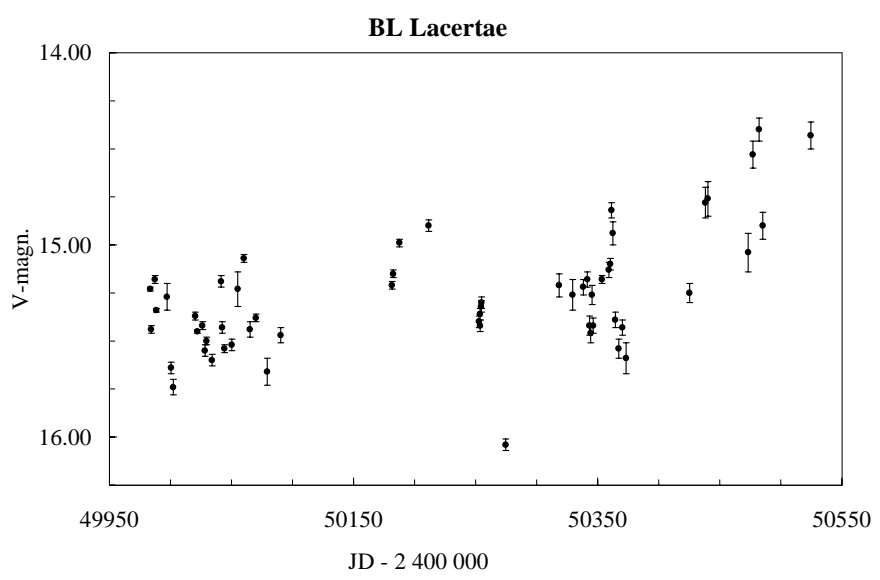

Fig. 20. Light curve of BL Lacertae

The minimum brightness was $15.02(\overline{\mathrm{JD}} 50360)$ and the maximum 14.23 ( $\overline{\mathrm{JD}} 50554)$.

\subsection{BL Lacertae}

BL Lacertae (PKS 2200+420) is the prototype of all BL Lac objects. Originally it was classified as a variable star in the 1920's. Its historical light curve shows a measured brightness of between 12.5 and $16.5 \mathrm{mag}$ in the $B$ band (Shen \& Usher 1970). The average brightness in the $V$-band has been between mag 14 and 15 (Webb et al. 1988; Moles et al. 1985; Sillanpää et al. 1988a).

Our light curve does not show (Fig. 20) any quiescent state at all. The brightness has fluctuated continuously, typically $0.2-0.3 \mathrm{mag}$ in a timescale of a few days. The maximum $V$-band brightness during our monitoring was 14.40 ( $\overline{\mathrm{JD}} 50482$ ) and the minimum 16.04 ( $\overline{\mathrm{JD}} 50274)$. The average brightness of BL Lacertae was rising during our observations between 1995 and 1997. It culminated in summer 1997 (after the observations presented here) when BL Lacertae had a large outburst (Webb et al. 1998). 


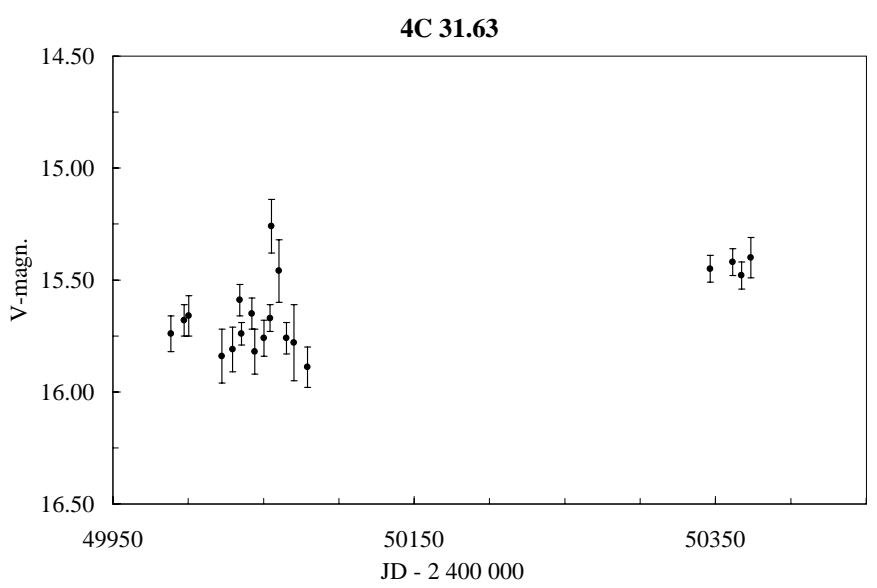

Fig. 21. Light curve of $4 \mathrm{C} 31.36$

\subsection{4. $4 C 31.36$}

4C 31.36 (QSO 2201+315) is a 4C-radio catalog object (Pilkington \& Scott 1965) and has been classified as a low polarization quasar, LPQ. It was first optically identified by Tritton et al. (1973). Its brightness has normally varied between mag 15 and 16 in the $B$-band (Tritton et al. 1973). Pica et al. (1988) reported variations of $0.77 \mathrm{mag}$.

During our monitoring (Fig. 21) 4C 31.36 had modest amplitude variations and only one possible major flare was recorded, a $0.41 \mathrm{mag}$ brightening on December 1995 ( $\overline{\mathrm{JD}} 50055)$. The maximum value of the brightness was 15.26 ( $\overline{\mathrm{JD}} 50055)$ and the minimum 15.89 ( $\overline{\mathrm{JD}} 50079)$. In general, excluding that one flare (only two datapoints), the variations of this object were small, which was expected for a low polarization quasar (LPQ).

\subsection{CTA 102}

CTA 102 (PKS 2230+11) is a high polarization quasar, HPQ (Sandage \& Wyndham 1965). Pica et al. (1988) reported $17.66 \mathrm{mag}$ as the long-term average brightness in the $B$-band and $1.14 \mathrm{mag}$ as the variability amplitude. It is also a strong $\gamma$-ray source (Blom et al. 1995; Thompson et al. 1995; Lin et al. 1996). Recent optical monitoring results, for example by Villata et al. (1997), and Raiteri et al. (1998), showed that the brightness variations of CTA 102 were small.

Our CTA 102 observations show one very large and rapid flare: $1.13 \mathrm{mag}$ on a timescale of 3 days (JD 50346) (Fig. 22 and Table 2). The 1.13 mag brightening is based on measurements from three separate images. More evidence for this very rapid and violent outburst can be found in the light curve by Tornikoski et al. (1999) who also detected the flare. The maximum $V$-band brightness during our observations was 15.23 ( $\overline{\mathrm{JD}} 50346)$ and the minimum $17.24(\overline{\mathrm{JD}} 50050)$.

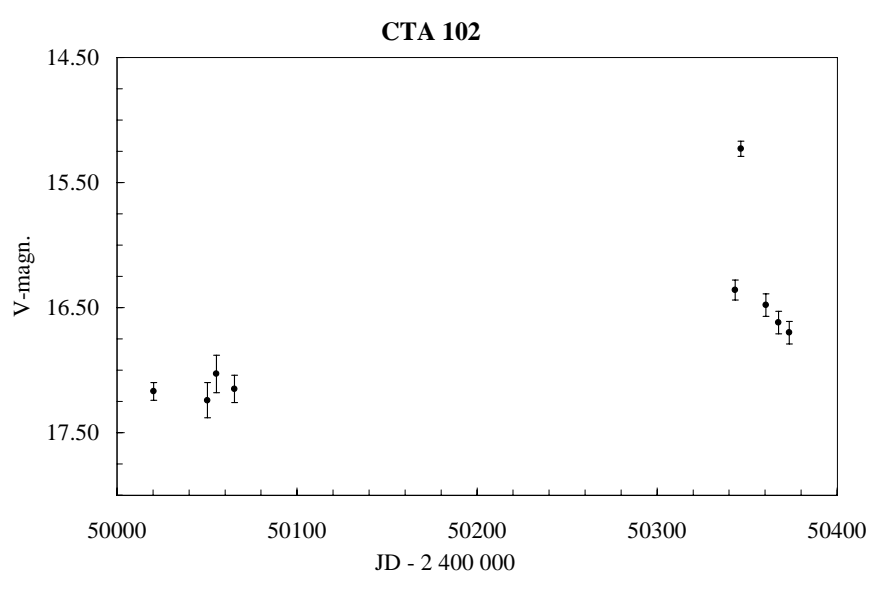

Fig. 22. Light curve of CTA 102

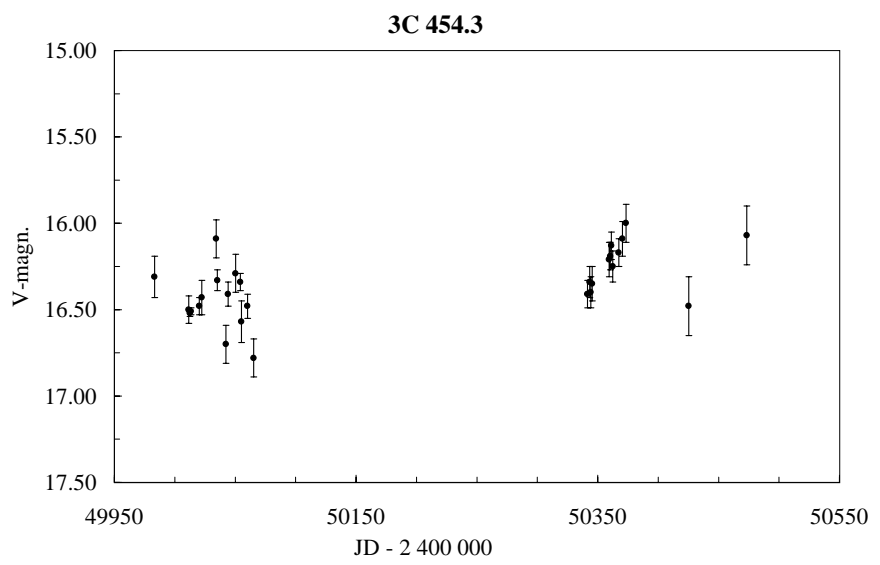

Fig. 23. Light curve of 3C 454.3

3.26. $3 C 454.3$

3C 454.3 (PKS 2251+15) is a strongly variable high polarization quasar, $\mathrm{HPQ}$. It can also be classified as an OVV quasar. Its $B$-band brightness has often been between mag 16 and 17 (Webb et al. 1988; Sillanpää et al. 1988a). The latest monitoring results are by Villata et al. (1997) and Raiteri et al. (1998). During those observations 3C 454.3 showed $0.4 \mathrm{mag}$ variations in the $R$-band, and only $0.12 \mathrm{mag}$ in the $V$-band. 3C 454.3 is a strong EGRET source (Blom et al. 1995; Thompson et al. 1995).

During our observations, the object showed frequent small amplitude variations (Fig. 23). The maximum $V$-band brightness was $16.00(\overline{\mathrm{JD}} 50373)$ and the minimum 16.78 ( $\overline{\mathrm{JD}} 50065)$.

\subsection{7. $O Y 091$}

OY 091 (PKS 2254+07) is a BL Lac object. Its historical brightness variations have been $1.6 \mathrm{mag}$ according to Pollock (1975). Pica et al. (1988) reported on 2.37 mag variations during 1.39 years. In $19811.8 \mathrm{mag}$ outburst was detected. After that flare the brightness faded 1.3 mag in 


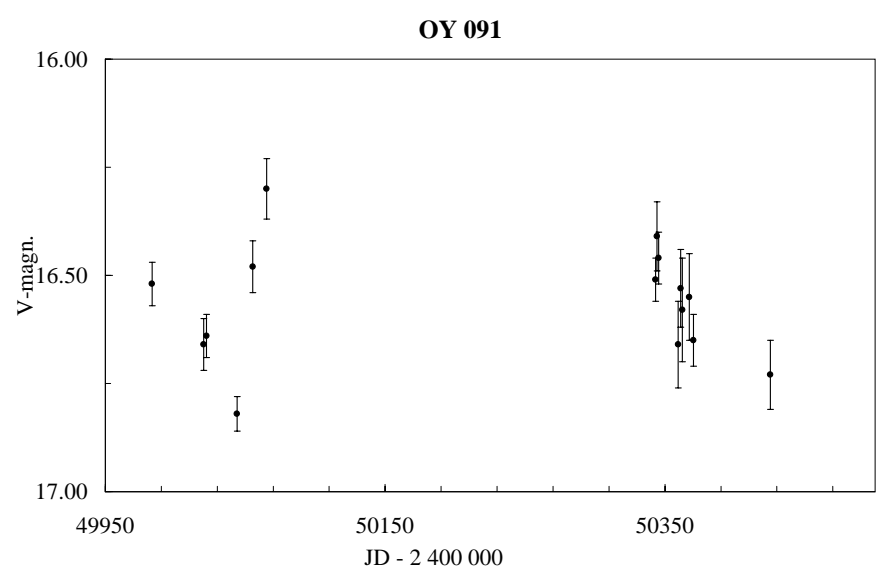

Fig. 24. Light curve of OY 091

18 days ( $B$-band) and continued fading to mag 18 where the brightness stayed for years (Pica et al. 1988).

During our observations the maximum $V$-band brightness value (Fig. 24) was 16.30 (JD 50065) and minimum 16.82 ( $\overline{\mathrm{JD}} 50044 \mathrm{mag})$. Due to the limited number of datapoints a more detailed analysis is not possible.

\section{Conclusions}

We have presented results of the Tuorla Optical Quasar Monitoring program from two observing seasons, from autumn 1995 to spring 1997. Our observations have shown that some blazars might have quite large flux variations in timescales of a few days, the best example being CTA 102. We found some indications for possible periodicity on a timescale of $60-70$ days in S5 0716+714 (not yet confirmed) and in 3C 66A (see Lainela et al. 1999) and observed the second peak of the predicted outburst in OJ 287. We measured one of the brightest $V$-band values ever for S2 0109+224, 3C 66A, and ON 231 and one of the faintest $V$-band values ever for OI 090.4.

Considering our sample of BL Lacs, HPQs, and LPQs we can say, as a conclusion, that those few LPQs, which were selected to our sample, did not show as large optical variations as BL Lacs or HPQs. No difference between BL Lacs and HPQs can be seen.

Despite the large amount of collected data, we must still say that more data is needed to get better understanding of the behaviour of these highly variable objects. Only long term monitoring of these blazars and quasars can show if the observed brightness variations are totally sporadic or not. The best examples of possible non-sporadic variations are blazars OJ 287 and 3C $66 \mathrm{~A}$. This emphasizes also the importance of collaboration between observatories which have similar monitoring programs.

Acknowledgements. This work was partly supported by the Academy of Finland.

\section{References}

Angel J.R.P., Stockmann H.S., 1980, ARA\&A 18, 321

Angione R.J., 1971, AJ 76, 412

Balonek T.J., 1998, http://quasar.colgate.edu/thalonek/ optical/CTA102compstars.gif

Barbieri C., Romano G., Di Serego S., Zambon M., 1977, A\&A 59,419

Baumert J.H., 1980, PASP 92, 156

Bertaud C., Dumortier B., Veron P., et al., 1969, A\&A 3, 436

Biermann P.L., Duebeck H., Eckart A., et al., 1981, ApJ 247, L53

Biraud F., 1971, Nat 232, 178

Blake G.M., 1970, ApJ 6, L201

Blom J.J., Bloemen H., Bennett K., et al., 1995, A\&A 295, 330

Bloom S.D., Bertsch D.L., Hartman R.C., 1997, ApJ 490, L145

Browne I.W.A., 1971, Nat 231, 515

Burbidge G., Hewitt A., 1989, in BL Lac Objects, Maraschi L., Maccacaro T., Ulrich M.H. (eds.). Springer Verlag, New York, p. 412

Catanese M., Akerlof C.W., Biller S.D., et al., 1997, ApJ 480, 562

Craine E., 1977, Handbook of Quasistellar and BL Lacertae Objects. Pachart Publishing House, Tuscon

Eachus L.J., Liller W., 1975, ApJ 200, L61

Fichtel C.E., Bertsch D.L., Chiang J., et al., 1994, ApJS 94, 551

Fiorucci M., Tosti G., 1996a, A\&AS 116, 403

Fiorucci M., Tosti G., 1996b, A\&AS 117, 475

Fiorucci M., Tosti G., Rizzi N., 1998, PASP 110, 105

Fleming T.A., Green R.F., Jannuzi B.T., 1993, AJ 106, 1729

Hall D.L., Usher P.D., 1972, Nat 241, 31

Heidt J., 1993, in "Proc. 1st MEGAPHOT Workshop Hamburg", Borgeest U., Schramm K.J., von Linde J. (eds.). Springer Verlag, p. 95

Impey C.D., Tapia S., 1988, ApJ 333, 666

Kidger M.R., Takalo L.O., 1990, A\&A 239, L9

Kinman T.D., 1976a, IAU Circ. 2908

Kinman T.D., 1976b, ApJ 205, 1

Kühr H., Witzel A., Pauliny-Toth I.I.K., Nauber U., 1981, A\&AS 45, 367

Kühr H., Johston K.J., Odenwald S., Aldhoch J., 1987, A\&AS 71,493

Lainela M., Takalo L.O., Sillanpää A., et al., 1999, ApJ 521, 561

Lin Y.C., Bertsch D.L., Dingus B.L., 1995, ApJ 442, L96

Lin Y.C., Bertsch D.L., Dingus B.L., et al., 1996, ApJS 105, 331

Liu F.K., Xoe G.Z., Bai J.M., 1995, A\&A 295, 1

Markarian B.Ye, Lipovetsky W.A., 1972, Astrofizika 8, 155

Massaro E., Nesci R., Maesano M., et al., 1996, A\&A 314, 87

Mattox J.R., Bertsch D.L., Chiang J., et al., 1993, ApJ 410, 609

McGimsey B.Q., Smith A.G., Scott R.I., et al., 1975, AJ 80

Miller H.R., 1985, IAUC 4076, 2

Miller H.R., Mullikin T.L., McGimsey B.Q., 1983, AJ 88, 1301

Moles M., Garcia-Pelayo J.M., Masegosa J., Aparicio A., 1985, ApJS 58, 255

von Montigny C., Bertsch D.L., Chiang J., et al., 1995, ApJ 440,525

Owen F.N., Mufson S.L., 1977, AJ 82, 776 
Pauliny-Toth I.I.K., Witzel A., Preuss E., et al., 1978, AJ 83, 451

Penston M.V., Cannon R.O., 1970, R. Obs. Bull. 159, 85

Petry D., Bradbury S.M., Konopelko A., et al., 1996, A\&A 311, L13

Pica A.J., 1977, AJ 82, 935

Pica A.J., Pollock J.T., Smith A.G., et al., 1980, AJ 85, 1442

Pica A.J., Smith A.G., Webb J.R., et al., 1988, AJ 96, 1215

Pilkington J.D.H., Scott P.F., 1965, MNRAS 69, 183

Pollock J.T., 1975, ApJ 198, L53

Pollock J.T., Pica A.J., Smith A.G., et al., 1979, AJ 84, 1658

Quirrenbach A., Witzel A., Kirchbaum T., et al., 1991, ApJ 372, L71

Raiteri C.M., Ghisellini G., Villata M., et al., 1998, A\&AS 127, 445

Raiteri C.M., Villata M., Tosti G., et al., 1999, A\&A 352, 19

Sandage A., 1967, ApJ 150, L177

Sandage A., Wyndham D., 1965, ApJ 141, 328

Schramm K.J., Borgeest U., Camenzind M., et al., 1993, A\&A 278,391

Schubnell M.S., Akerlof C.W., Biller S., et al., 1996, ApJ 460, 644

Shen B.S.P., Usher P.D., 1970, Nat 228, 1070

Sillanpää A., Haarala S., Korhonen T., 1988a, A\&AS 72, 347

Sillanpää A., Haarala S., Valtonen M.J., Sundelius B., Byrd G.G., 1988b, ApJ 325, 628

Sillanpää A., Mikkola S., Valtaoja L., 1991, A\&AS 88, 225

Sillanpää A., Takalo L.O., Pursimo T., et al., 1996a, in "Workshop on two years of intensive monitoring of OJ 287 and 3C 66A", Takalo L.O. (ed.), Tuorla Observatory Reports No. 176,7

Sillanpää A., Takalo L.O., Pursimo T., et al., 1996b, A\&A 305, L17

Sillanpää A., Takalo L.O., Pursimo T., 1996c, A\&A 315, L13

Smith P.S., Balonek T.J., Heckert P.A., et al., 1985, AJ 90

Smith P.S., Balonek T.J., Heckert P.A., et al., 1987, ApJS 64, 459

Takalo L.O., 1991, A\&AS 90, 161

Takalo L.O., 1994, VA 38, 77
Takalo L.O., Sillanpää A., Nilsson K., 1994, A\&AS 107, 497

Takalo L.O., Sillanpää A., Pursimo T., et al., 1996, A\&AS 120, 313

Takalo L.O., Sillapää A., Valtaoja E., et al., 1998, A\&AS 129, 577

Tapia S., Craine E., Gearhart M.R., et al., 1977, ApJ 215, L71

Teräsranta H., Valtaoja E., 1996, A\&AS 120, 491

Thompson D.J., Bertsch D.L., Dingus B.L., et al., 1995, ApJS 101,259

Tornikoski M., Teräsranta H., Balonek T.J., Beckerman E., 1999, in "BL Lac Phenomenon", Takalo L.O., Sillanpää A. (eds.), PASP 159, 307

Tosti G., Fiorucci M., Luciani M., et al., 1998a, A\&A 339, 41

Tosti G., Fiorucci M., Luciani M., et al., 1998b, A\&AS 130, 109

Tritton K.P., Henbest S.N., Penston M.V., 1973, MNRAS 162, 31

Véron M.P., Véron P., 1973, A\&A 28, 319

Véron P., Véron M.P., 1975, A\&A 39, 281

Véron P., Véron M.P., 1976, A\&AS 25, 287

Villata M., Raiteri C.M., Ghisellini G., et al., 1997, A\&AS 121, 119

Wagner S.J., 1996, A\&AS 120, 495

Wagner S.J., Witzel A., Krichbaum T.P., et al., 1993, A\&A 271,344

Wagner S.J., Witzel A., Heidt J., et al., 1996, AJ 111, 2187

Webb J.R., Smith A.G., Leacock R.J., et al., 1988, AJ 95, 374

Webb J.R., Carini M.T., Clements S., et al., 1990, AJ 100, 1452

Webb J.R., Bernello T., Robson T., Hartman R.C., 1995, IAUC 6168

Webb J.R., Freedman I., Howard E., et al., 1998, AJ 115, 2244

Wehrle A.E., Pian E., Urry C.M., et al., 1998, ApJ 497, 178

Wills B.J., Wills D., 1974, ApJ 190, L97

Wills B.J., Pollock J.T., Aller H.D., et al., 1983, ApJ 274, 62

Xie G.Z., Li K.H., Zhang Y.H., et al., 1994, A\&AS 106, 361

Zekl H., Klare G., Appenzeller I., 1981, A\&A 103, 342

Zwicky F., 1966, ApJ 143, 192 